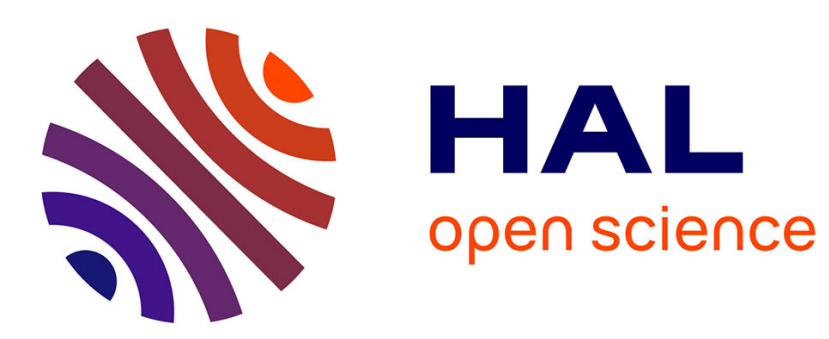

\title{
Few-cycle optical solitons in linearly coupled waveguides
}

\author{
Said Terniche, Hervé Leblond, Dumitru Mihalache, Abdelhamid Kellou
}

\section{To cite this version:}

Said Terniche, Hervé Leblond, Dumitru Mihalache, Abdelhamid Kellou. Few-cycle optical solitons in linearly coupled waveguides. Physical Review A, 2016, 94 (6), pp.063836. 10.1103/PhysRevA.94.063836 . hal-02572840

\section{HAL Id: hal-02572840 \\ https://univ-angers.hal.science/hal-02572840}

Submitted on 13 May 2020

HAL is a multi-disciplinary open access archive for the deposit and dissemination of scientific research documents, whether they are published or not. The documents may come from teaching and research institutions in France or abroad, or from public or private research centers.
L'archive ouverte pluridisciplinaire HAL, est destinée au dépôt et à la diffusion de documents scientifiques de niveau recherche, publiés ou non, émanant des établissements d'enseignement et de recherche français ou étrangers, des laboratoires publics ou privés. 


\title{
Few-cycle optical solitons in linearly coupled waveguides
}

\author{
Said Terniche, ${ }^{1,2}$ Hervé Leblond, ${ }^{1}$ Dumitru Mihalache, ${ }^{3,4}$ and Abdelhamid Kellou ${ }^{2}$ \\ ${ }^{1}$ LUNAM Université, Université d'Angers, Laboratoire de Photonique d'Angers, EA 4464, 2 Boulevard Lavoisier, F-49000 Angers, France \\ ${ }^{2}$ Laboratoire Electronique Quantique, USTHB, BP 32 El-Alia, 16011 Bab Ezzouar Alger, Algérie \\ ${ }^{3}$ Academy of Romanian Scientists, 54 Splaiul Independentei, RO-050094 Bucharest, Romania \\ ${ }^{4}$ Horia Hulubei National Institute for Physics and Nuclear Engineering, 30 Reactorului, Bucharest-Magurele, RO-077125, Romania
}

(Received 13 June 2016; published 16 December 2016)

\begin{abstract}
We consider soliton propagation in two parallel optical waveguides, in the presence of a linear nondispersive coupling and in the few-cycle regime. The numerical analysis is based on a set of two coupled modified Korteweg-de Vries equations. The evidenced few-cycle vector solitons are optical breathers. In addition to the usual breathing due to carrier-envelope velocity mismatch, we observe, and describe in detail, spatial oscillations of soliton's amplitude and energy.
\end{abstract}

DOI: 10.1103/PhysRevA.94.063836

\section{INTRODUCTION}

During the past years the use of ultrashort, few-cycle intense laser pulses extended the capability of observing and controlling unique physical phenomena, such as electron motion in atoms, molecular vibrations, and chemical reactions of complex molecules; see the review [1]. The intense experimental and theoretical activity in this area followed the seminal experimental results that were reported in 1999 by different research groups [2-5], on the generation and characterization of ultrashort (two-cycle or even sub-two-cycle) optical pulses from Kerr-lens mode-locked Ti:sapphire lasers. Such few-cycle intense optical pulses can be used in a variety of applications ranging from light-matter interactions, highorder harmonic generation, extreme nonlinear optics [6,7], attosecond physics $[8,9]$, and open the door to zeptosecondexawatt physics (i.e., the possibility of compression of petawatt pulses into the exawatt and zeptosecond regime) [10-12].

On the theoretical arena, a lot of studies in the area of ultrashort light pulses were based on the slowly varying envelope approximation (SVEA) by using various generalizations of the nonlinear Schrödinger (NLS) equation; see, for example, Refs. [13,14]. In addition, various theoretical approaches have been put forward beyond the common SVEA, such as the so-called unidirectional pulse propagation model $[15,16]$, the Maxwell-Duffing description of ultrashort optical pulses in nonresonant media [17], and the Maxwell-Drude-Bloch model of few-cycle optical solitons [18]; for recent overviews of the main theoretical approaches in the area of nonlinear optics of ultrashort light pulses including few-cycle optical solitons, see Refs. [19-22]. The generic theoretical models beyond the SVEA that describe the evolution of such intense electric fields rely on the modified Korteweg-de Vries (mKdV) [23], the short-pulse [24-26], the sine-Gordon (sG) [27], double sG $[8,28]$, and the $\mathrm{mKdV}$-sG [29-31] equations; see also Refs. [32-36]. Recent relevant works in this very broad area deal with the study of subcycle optical breathing solitons [37], the problem of extreme self-compression of few-cycle optical solitons in the ionization regime [38], the generation of unipolar optical pulses in a Raman-active medium [39], the compression and collisions of chirped pulses in two-level media [40], the study of interplay of diffraction and nonlinear effects in the propagation of ultrashort pulses [41], and the dynamics of few-cycle solitons in birefringent media containing both symmetrical and asymmetrical molecules [42]. Although most of the above mentioned generic models are one-dimensional (1D) ones, studies of multidimensional fewcycle optical solitons have been also performed. It was shown in Ref. [43] that in bulk media, depending on the sign of the nonlinear coefficient, the cubic (Kerr-type) nonlinearity can lead to either catastrophic self-focusing or the enhancement of the diffraction effect. However, in quadratic nonlinear media either stable plane waves or stable few-cycle spatiotemporal optical solitons (alias light bullets) can form from few-cycle input pulses [44]; for a few papers that overview both the experimental and theoretical activity in the area of linear and nonlinear light bullets in a series of relevant physical settings see Refs. [45-48].

Typical 1D media supporting localized light propagation are planar waveguides and cylindrical optical fibers that rely on either refractive index variations of adjacent dielectric media or the special physical features of photonic crystals. The experimental studies of supercontinuum generation in photonic crystal fibers [49] are intimately related to the associated problem of the propagation of few-cycle pulses in such media [50,51].

Although the problem of few-cycle soliton propagation in optical media motivated a lot of theoretical studies, few of them considered the transverse evolution. However, the huge intensities required for few-cycle soliton formation require that pulses are strongly localized in both time and space. In bulk media, they may collapse or be strongly diffracted [43]. Hence, studies of propagation of few-cycle pulses in optical waveguides are important from both theoretical and experimental point of view. We mention here that theoretical studies of the propagation of few-cycle pulses in coupled optical waveguides have been performed by using the unidirectional pulse propagation equation [52] and experimental results on ultrashort pulse propagation in arrays of subwavelength waveguides have also been reported [53].

We considered some time ago [54] the waveguiding problem of few-cycle pulses in the frame of mKdV-type models, and we showed that nonlinear coupling can strongly modify the characteristic waveguiding properties. More recently, we established the generic equations that account for the coupling between two adjacent optical waveguides [55]. The analysis 
was performed in the frame of a non-SVEA model, namely the generalized Kadomtsev-Petviashvili equation and a set of two coupled mKdV equations was derived [55]. It was revealed that three types of coupling can occur, involving the linear index, the dispersion, or the nonlinearity. The extensive numerical simulations involving a linear nondispersive coupling presented in Ref. [55] show a quite complex interaction between incident pulses. In order to describe properly this interaction, and to predict the outcomes of the optical coupler, it is necessary to determine and to describe as accurately as possible the stationary states, i.e., the vector solitons of the coupled system. This is the goal of the present paper. We will see that the typical oscillation between the two channels, which is known to occur in the linear regime, still exists in the case of few-cycle solitons, but with different frequency and amplitude.

The organization of this paper is as follows. In Sec. II we present the generic model based on the coupled $\mathrm{mKdV}$ equations and we discuss the key features of the corresponding few-cycle vector solitons. The detailed study of the amplitude and energy oscillations of the few-cycle vector solitons is given in Sec. III. Finally, Sec. IV concludes this paper.

\section{THE MODEL AND THE FEW-CYCLE VECTOR SOLITONS}

The governing model is the set of coupled $\mathrm{mKdV}$ equations,

$$
\begin{aligned}
& \partial_{z} u+a \partial_{t}\left(u^{3}\right)+b \partial_{t}^{3} u+c \partial_{t} v=0 \\
& \partial_{z} v+a \partial_{t}\left(v^{3}\right)+b \partial_{t}^{3} v+c \partial_{t} u=0
\end{aligned}
$$

Here $t$ and $z$ are the normalized time and propagation distance, respectively, whereas $u$ and $v$ are the normalized components of the few-cycle vector soliton.

It is very unlikely that the system of coupled mKdV Eqs. (1) and (2) admit any analytical solution, except in the special case where the system reduces to a single $\mathrm{mKdV}$, i.e., if $u= \pm v$. In what follows we give some reasonable arguments supporting this conclusion. It is worth mentioning that no analytical periodic solution, either localized or periodic, of the nonintegrable system (1) and (2) is known, to the best of our knowledge. The Hirota bilinear form [56] of the $\mathrm{mKdV}$ equation cannot be extended to the system (1) and (2). Indeed, the Hirota method involves the transform $u=\partial_{x}\left[C \arctan \left(\frac{g}{f}\right)\right]$ (with adequately chosen constant $C$ ), which lets appear the denominator $f^{2}+g^{2}$ in the $u$ component of the sought-after exact solution. An analogous transform for $v$ as $v=\partial_{x}\left[D \arctan \left(\frac{h}{k}\right)\right]$ would make appear another independent denominator $h^{2}+k^{2}$ in the $v$ component of the sought-after exact solution, which would prevent from deriving a coupled Hirota bilinear form. However, several works were devoted to the study of analytic solutions to different forms of coupled mKdV-type equations. Apart from the so-called complex mKdV equation [57], in most of them the coupling is purely nonlinear, and in many cases noncoupling nonlinear terms were not considered; see, e.g., Refs. [58,59]. A very general system of coupled $\mathrm{mKdV}$ equations was presented in Ref. [60], of which our system (1) and (2) is a special case if we assume that all the coefficients $r_{j}$ and $e_{j}$ of the coupled $\mathrm{mKdV}$ equations introduced in Ref. [60] are zero excepting $r_{1}=e_{1}, r_{2}=e_{2}$, and $r_{12}=e_{12}$, which coincide with our coefficients $b, 3 a$, and $c$, respectively. In Ref. [60] three different kinds of periodic cnoidal-type solutions to the coupled $\mathrm{mKdV}$ system were reported. It is easily checked that the conditions for the first solution reported in Ref. [60] are not satisfied in our case. Regarding the second type of solution given in Ref. [60], the analysis is not as obvious, however, it can be shown that, excepting the case when the two coupled fields $u$ and $v$ coincide, for which the coupled system reduces to a single $\mathrm{mKdV}$ equation, the solvability conditions always reduce to $a=b=0$, i.e., the resulting equation is the trivial one. The third kind of solution given in Ref. [60] is that corresponding to the reduction of the coupled system to a single $\mathrm{mKdV}$ equation, when $u$ and $v$ are proportional. Hence, according to the results reported in Ref. [60], our coupled nonlinear system does not admit any type of periodic cnoidal wave solutions, and it is very unlikely that any other periodic solution could be found analytically. The above considerations do not constitute a proof of the fact that no analytical solution of the system (1) and (2) exist at all (excepting the case $u= \pm v$ ). However, the arguments presented above constitute a strong indication that this conclusion holds.

In this work we solve numerically the set of coupled $\mathrm{mKdV}$ Eqs. (1) and (2) by means of a standard fourth-order RungeKutta scheme, in which the $t$ derivatives are computed using five-point finite difference formulas.

The transformation of variables,

$$
t \longmapsto t \sqrt{\frac{c}{b}}, \quad z \longmapsto z c \sqrt{\frac{c}{b}}, \quad\left(\begin{array}{l}
u \\
v
\end{array}\right) \longmapsto \sqrt{\frac{a}{2 c}}\left(\begin{array}{l}
u \\
v
\end{array}\right),
$$

reduces Eqs. (1) and (2) to the situation where $a=2$ and $b=c=1$. We use these normalized values of the coefficients throughout the paper. The above transformation of variables (3) exists provided that $a, b$, and $c$ have the same sign. The condition $a b>0$ means that the nonlinearity leads to pulse compression; this assumption is required for the formation of solitons. However, the condition $b c>0$ is not restrictive; the transformation

$$
\left(\begin{array}{l}
u \\
v
\end{array}\right) \longmapsto\left(\begin{array}{r}
u \\
-v
\end{array}\right)
$$

implies the change of the sign of $c$ in Eqs. (1) and (2). Further, we will operate at some fixed frequency and energy.

The stationary states are not constant but oscillate with the propagation distance $z$. Generally speaking, few-cycle solitons are breathers; further we will see that the peak values and durations of both pulse components of the stable states oscillate with $z$. For convenience, we will still call them "stationary states" in spite of this intrinsic feature. Due to this peculiar feature, we cannot use the standard procedures to compute the stationary states, but need to extract them from the evolution equation. We use the following procedure: We start from some arbitrary initial data not too far away from the stationary state we want to obtain. As a first trial, we take for $u$ the analytical breather solution of the $\mathrm{mKdV}$ equation, i.e., of Eq. (1) with $c=0$. The analytical solution initially derived in [56] can be found, e.g., in [27] (we use the parameter $p_{1}=1+4 i$, with the notations of [27]). For the second component we set $v=q u$ with $q$ some real constant. A previously computed soliton 


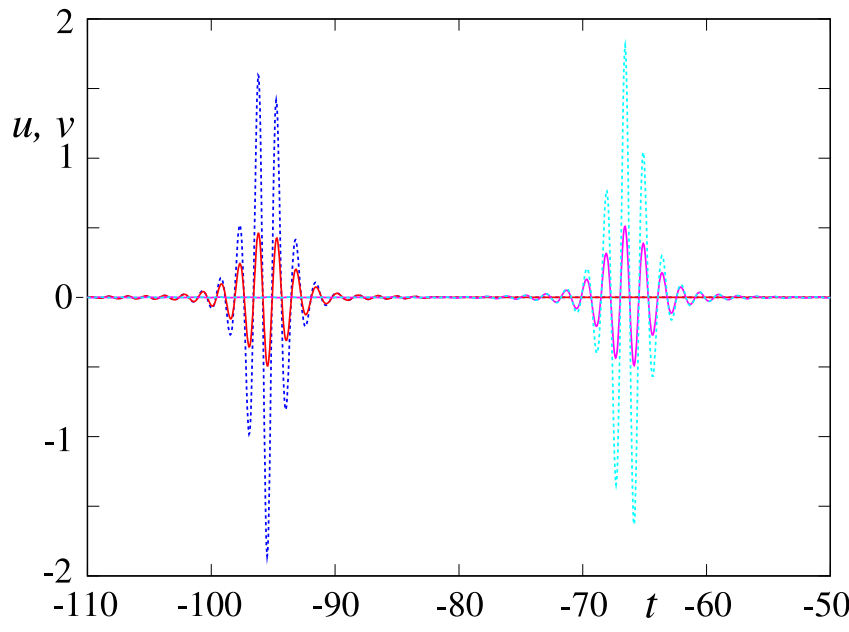

FIG. 1. Example of soliton. $u$, dotted; $v$, solid; left, at $z=0$; right, at $z=60$. Soliton with $\left\langle A_{u}\right\rangle=1.837$.

slightly modified by some arbitrary perturbation can also be used. Then we run the evolution until a soliton is formed and separated from the so-called radiation. The radiation is removed by temporal filtering, i.e., $u$ and $v$ are set to zero before and after the pulse, stopping at or starting from some fixed time from the center of the pulse, and the obtained result is used as a new input for propagation. The procedure is repeated until the pulse does not radiate any continuous wave any more.

Figure 1 gives a typical example of few-cycle vector soliton. In addition, the symmetry of the equation shows that the transform $(u, v) \longrightarrow(v, u)$ leave the system unchanged, and consequently a second family of solitons straightforwardly deduces from the considered one.

Figure 2 shows the evolution of soliton's maximum amplitude during propagation. We observe that the solitons feature two types of oscillations: The faster one is the usual breathing of the few-cycle pulses, which corresponds to the mismatch between phase and group velocities. The slower one
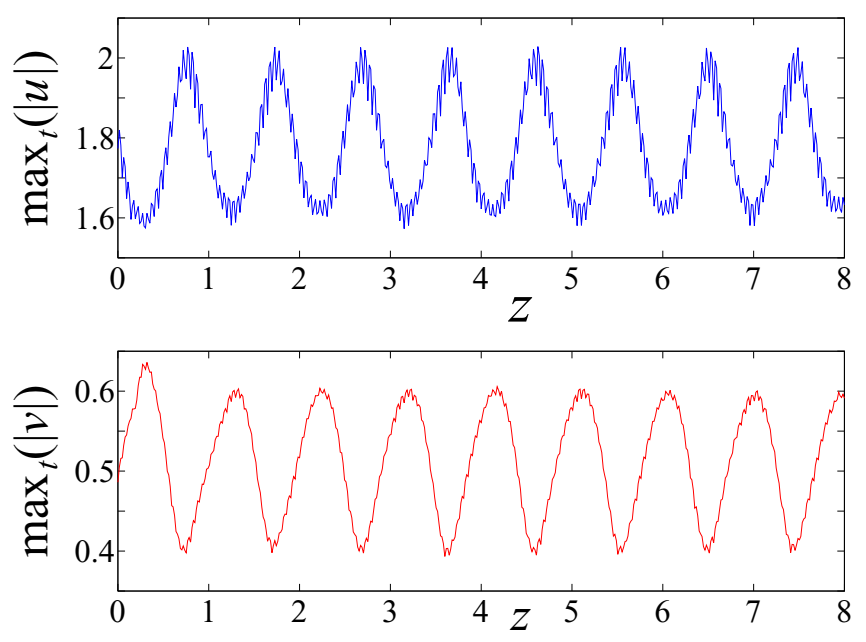

FIG. 2. Evolution of the soliton amplitude $\max _{t}(|u|)$ (top) and $\max _{t}(|v|)$ (bottom). Soliton with $\left\langle A_{u}\right\rangle=1.789$. corresponds to a periodic energy exchange between the two channels, as it exists in the linear situation.

The linearized version of the coupled Eqs. (1) and (2), i.e., when $a=0$, is easily solved for monochromatic plane waves. The plane wave solutions of the uncoupled equations are

$$
\left(\begin{array}{l}
u \\
v
\end{array}\right)=\left(\begin{array}{l}
A \\
B
\end{array}\right) e^{-i\left(\omega t+b \omega^{3} z\right)},
$$

and when the coupling is introduced, the amplitudes $A$ and $B$ depend on $z$ according to

$$
\begin{aligned}
& A=u_{0} \cos c \omega z+i v_{0} \sin c \omega z, \\
& B=v_{0} \cos c \omega z+i u_{0} \sin c \omega z,
\end{aligned}
$$

and consequently the maximum amplitude and the power density of the wave oscillate with spatial frequency $c \omega / \pi$.

We next define the central frequency of the soliton as

$$
v_{0}=\frac{\int_{0}^{\infty} v \hat{u}(v)^{2} d v}{\int_{0}^{\infty} \hat{u}(v)^{2} d v},
$$

where $\hat{u}$ is the Fourier transform of $u$.

The central frequency of all the computed solitons, evaluated using (8), remains very close to the value of $v_{0}=0.663$ obtained for the $\mathrm{mKdV}$ breather with parameter $p_{1}=1+4 i$ with which we started the analysis.

The maximum amplitude and energy of monochromatic waves with frequency $v_{0}$ oscillate with spatial frequency $2 c v_{0}=1.326$. The spatial oscillation frequencies $\sigma_{a}$ that are obtained by analyzing the energy and amplitude oscillations of the solitons have the same order of magnitude, but slightly lower: They range from 1.066 to 1.166 , increasing slightly with the soliton amplitude.

\section{THE AMPLITUDE AND ENERGY OSCILLATIONS OF THE FEW-CYCLE VECTOR SOLITONS}

The oscillations of the energies $E_{u}=\int u^{2} d t$ and $E_{v}=$ $\int v^{2} d t$ are almost perfectly harmonic, adequately fitted by expressions of the form

$$
E_{u}=\left\langle E_{u}\right\rangle+\Delta E_{u} \sin \left(2 \pi \sigma_{a} z+\phi_{E, u}\right),
$$

where $\left\langle E_{u}\right\rangle$ is the mean value and $\Delta E_{u}$ some amplitude, and analogously for $E_{v}$. In Eq. (9), $\sigma_{a}$ is the spatial oscillation frequency. Due to energy conservation, $\Delta E_{v}=\Delta E_{u}$, and the energies in the two parallel waveguides oscillate in phase opposition. The same holds for the maximum amplitudes $A_{u}=\max _{t}(|u|)$ and $A_{v}=\max _{t}(|v|)$, for which we can give expressions analogous to (9), with analogous notations. The spatial oscillation frequency $\sigma_{a}$ ranges from 1.06 to 1.17 , increasing approximately linearly with $\left\langle A_{u}\right\rangle$.

The values of $\Delta A_{u}, \Delta A_{v}$, and $\Delta E_{u}$ are extracted from the numerical data using the variance formula, as $\Delta E_{u}=$ $\sqrt{2\left(\left\langle E_{u}^{2}\right\rangle-\left\langle E_{u}\right\rangle^{2}\right)}$, where $\langle\cdot\rangle$ holds for averaging over $z$, and so on. We check that expression (9) adequately fits the $z$ evolution of the energy and amplitudes. They are plotted against $\left\langle A_{u}\right\rangle$ in Fig. 3.

The curves are well fitted with curves of the form

$$
\Delta E_{u} \simeq R \sqrt{A_{0}-\left\langle A_{u}\right\rangle},
$$




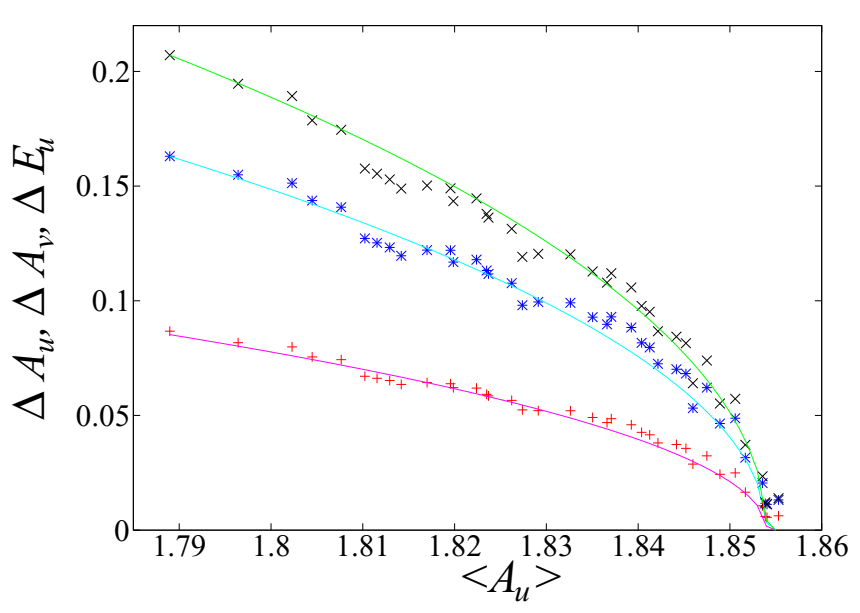

FIG. 3. The amplitude of the oscillations of the energy of $u\left(\Delta E_{u}\right.$, black crosses), of the maximum amplitude of $u$ ( $\Delta A_{u}$, blue stars), and of the maximum amplitude of $v\left(\Delta A_{v}\right.$, red crosses), against the mean value of the maximum amplitude of $u\left(\left\langle A_{u}\right\rangle\right)$. Solid lines are parabolic fits.

and so on, with the same value of $A_{0}=1.854$ in every case, which implies that $\Delta A_{u}, \Delta A_{v}$, and $\Delta E_{u}$ are proportional. In fact, the ratio $A_{u} / A_{v}=1.91 \pm 0.04$, the main fluctuations being due to the accuracy at the point where the oscillations vanish. The parabolic shape of these curves, the fact that the amplitude of the oscillations start from zero at the vertex, recalls to a Hopf bifurcation. However, the situation is different, since there is no external control parameter here. We were not able to evidence a solution with amplitude larger than $A_{0}$, which would not oscillate as we can guess from Fig. 3. Such solution can either be unstable, or nonexistant.

The pulse duration also evolves periodically with the propagation distance $z$, and differs for the two components $u$ and $v$. We evaluate the durations $T_{u}$ and $T_{v}$ from numerical data using the second-order momentum,

$$
T_{u}^{2}=C_{n}^{2} \frac{\int_{-\infty}^{\infty}\left(t-t_{u}\right)^{2} u^{n} d t}{\int_{-\infty}^{\infty} u^{n} d t},
$$

in which

$$
t_{u}=\frac{\int_{-\infty}^{\infty} t u^{n} d t}{\int_{-\infty}^{\infty} u^{n} d t}
$$

is the pulse center, and analogously for $v$. However, the standard value of the exponent, $n=2$, cannot be used because the contribution of the remaining radiation induces excessive numerical errors. Consequently, we use $n=4$ for $u$ and $n=8$ for $v$. The coefficients are defined in such a way that, for an hyperbolic secant pulse $u=A \operatorname{sech} p t, T_{u}=1 / p$. Straightforward computation of the integrals gives

$$
C_{4}=\frac{2 \sqrt{3}}{\sqrt{\pi^{2}-6}}, \quad C_{8}=\frac{6 \sqrt{2}}{\sqrt{6 \pi^{2}-49}} .
$$

We find that the durations $T_{u}$ and $T_{v}$ oscillate in time, and can be fitted by sine curves analogous to (9). For the largest component $u$, the duration $T_{u}$ and the maximum amplitude $A_{u}$ oscillate in phase opposition, so that the product is almost constant. It still oscillates, however, with amplitudes ranging from 0.012 to 0.09 , and mean value close to 2 .

It must be recalled that the $\mathrm{mKdV}$ equation, i.e., one of Eq. (1) or (2) with vanishing coupling $c=0$, admits breather solutions that can be interpreted as few-cycle solitons, and that some carrier wave and envelope can be defined for these breathers from the analytical expression of the solution and without any reference to the SVEA [29].

The expression of the envelope is the same as the one of the NLS soliton obtained within the SVEA and prolongates it in the few-cycle regime. It writes as

$$
u=2 \sqrt{\frac{2 b}{a}} p \operatorname{sech} p\left(t-\frac{z}{V}\right),
$$

where $p$ is an arbitrary parameter and $V$ is the group velocity of the pulse. In our computations $2 \sqrt{\frac{2 b}{a}}=2$, and the area of the soliton is $A_{u} \times T_{u}=2$. Hence the variations of the duration of the largest component $u$ of the vector soliton are correlated with the variations of amplitude in such a way that they satisfy the area theorem in the same way as in the absence of coupling. However, the cancellation is far from being exact. Multiplying $A_{u}=\left\langle A_{u}\right\rangle+\Delta A_{u} \sin (k z)$ and $T_{u}=\left\langle T_{u}\right\rangle-\Delta T_{u} \sin (k z)$, and writing the condition for the term proportional to $\sin (k z)$ to vanish, we get $\left\langle T_{u}\right\rangle \Delta A_{u}=\left\langle A_{u}\right\rangle \Delta T_{u}$. However, this condition is not satisfied, but $\left(\left\langle T_{u}\right\rangle \Delta A_{u}\right) /\left(\left\langle A_{u}\right\rangle \Delta T_{u}\right) \sim 0.7$. The oscillations of the durations are larger than what is required for the exact cancellation of this term.

The situation is completely different for the smaller $v$ component, since $T_{v}$ and $A_{v}$ oscillate in phase. The amplitude $A_{v}$ must decrease as $A_{u}$ increases due to energy conservation. The duration $T_{v}$ is driven by the duration $T_{u}$ and increases with it. As a result, $A_{v}$ and $T_{v}$ are in phase. Consequently the oscillations of their product are maximized. The amplitudes $\Delta T_{v}$ of the oscillations of $T_{v}$ follow the same square root law (10) as $\Delta E_{u}, \Delta A_{u}$, and $\Delta A_{v}$, as $\Delta T_{u}$ does. Notice that $\Delta T_{v}$ is quite larger than $\Delta T_{u}$ : The ratio $\Delta T_{v} / \Delta T_{u}$ increases from about 1.6 to about 1.8 as $\left\langle A_{u}\right\rangle$ increases in the range of the computed solitons.

If we assume that $u$ and $v$ are proportional, as $u=$ $A f(z, t), v=B f(z, t)$, where $A$ and $B$ are two constants, it is easily seen that Eqs. (1) and (2) reduce to a single $\mathrm{mKdV}$ equation to be satisfied by $f$ provided that $A= \pm B$, i.e., $u= \pm v$ (and using an adequate Galilean transform). We checked numerically that, at least for the value of parameters considered, this solution is unstable: The pulse turns into a vectorial soliton of the above family. Excepting this special unstable case, any other soliton solution requires a nontrivial relation between $u$ and $v$.

We want to see now how the ratio $v / u$ evolves, depending on diverse variables, and consider first the evolution of the angle $\theta=\arctan (v / u)$ with respect to $t$ along the pulse.

Figures 4 and 5 show a typical soliton profile. For completeness, the angle $\theta^{\prime}$ between the $u$ axis and the vector $(u, v)$ in the $(u, v)$ plane is also shown in Fig. 5. It is seen that the angle $\theta$ is close to a constant, although it is not exactly the case. The value of this constant can be computed for each propagation distance. It evolves periodically with $z$, with the same oscillation frequency as that corresponding to oscillations of energy, maximum amplitude, and duration 


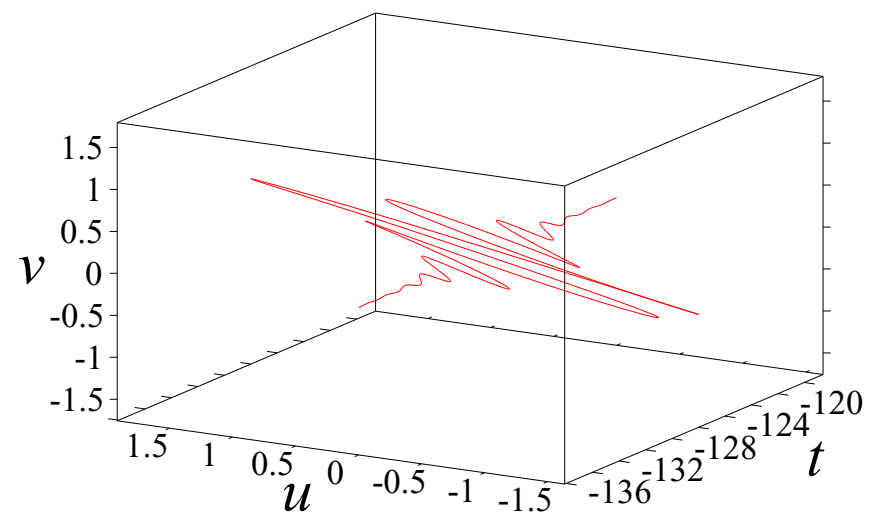

FIG. 4. A typical soliton profile in the $(u, v)$ plane vs time $t$. Soliton with $\left\langle A_{u}\right\rangle=1.855$.

according to an expression analogous to (9), with some mean value $\langle\theta\rangle$ and amplitude $\Delta \theta$, as we have checked with the same method as above. The oscillations of $\theta$ are in phase with the ones of $u$, and consequently in phase opposition with respect to $v$. The mean value $\langle\theta\rangle$ and amplitude $\Delta \theta$ of the angle can be plotted against the mean maximum amplitude $\left\langle A_{u}\right\rangle$ (see Fig. 6); the obtained curve is comparable to the curves of Fig. 3 , and can be fitted by a parabola according to Eq. (10), with the same vertex. As $\left\langle A_{u}\right\rangle$ increases, it is seen that $\Delta \theta$ decreases to zero from about one-fourth of the value of $\langle\theta\rangle$. In the linear case, the angle $\theta$ can be defined in the same way. It can be seen from Eqs. (6) and (7) that in the linear case the mean value is always $\langle\theta\rangle=45^{\circ}$ and the amplitudes $\Delta \theta$ of its oscillations can vary between $0^{\circ}$ and $90^{\circ}$. We thus conclude that the nonlinear behavior strongly differs from the linear one.

The oscillations of the angle $\theta$ are related to the correlation between the amplitudes of $u$ and $v$ imposed by the conservation of energy. Let us evaluate the effect of this correlation by a simple computation. We assume that $u=A(z) f(z, t)$ and $v=B(z) f(z, t)$ are proportional to some fixed profile $f$ (this is

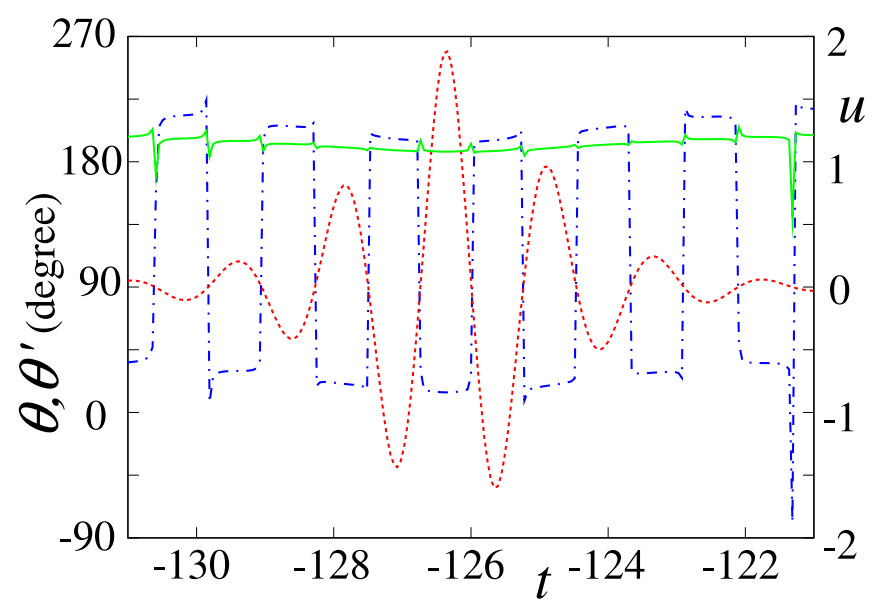

FIG. 5. The ratio between the two components $u$ and $v$ is shown using the angle $\theta=\arctan (v / u)$ (green solid line) vs time $t$. The profile of the component $u$ is given for clarity (red dotted line), and also the angle $\theta^{\prime}$ (blue dash-dotted line) between the $u$ axis and the vector $(u, v)$ in the $(u, v)$ plane. Soliton with $\left\langle A_{u}\right\rangle=1.855$.

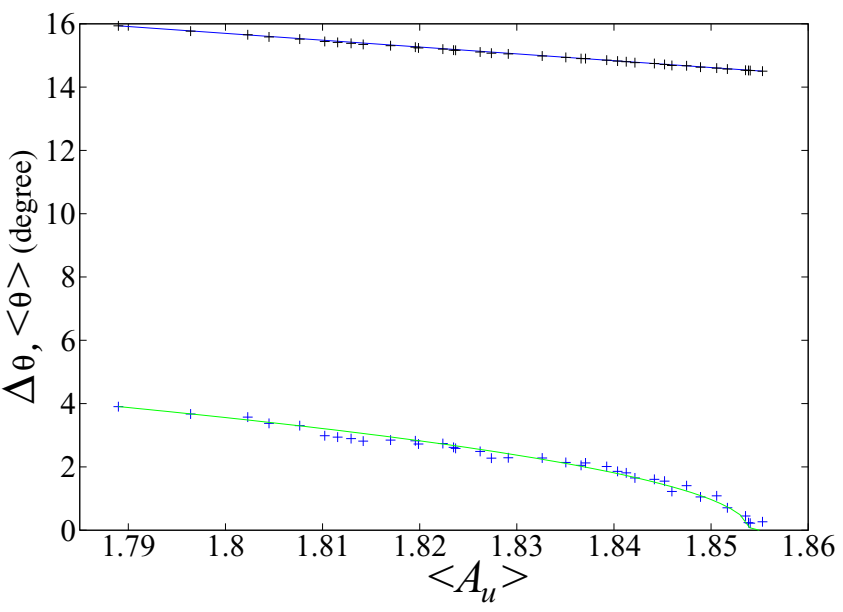

FIG. 6. The mean value $\langle\theta\rangle$ (black line on top of the figure) of the angle that measures the ratio between the two components $u$ and $v$, and the amplitude $\Delta \theta$ of its oscillations (green line on the bottom of the figure) are shown as a function of the mean value $\left\langle A_{u}\right\rangle$ of the maximum amplitude of $u$. The crosses indicate the results of numerical computation and the solid lines are linear or parabolic fits.

not possible unless $A \equiv \pm B$ ), and that the amplitude oscillates as $A(z)=A_{0}+\Delta A \cos k z$, and analogously for $B$, with $\Delta A$ and $\triangle B$ small with respect to $A_{0}$ and $B_{0}$. Conservation of the energy imposes that $B(z)$ and $A(z)$ evolve in phase opposition, i.e., $B(z)=B_{0}+\Delta B \cos k z$, with $A_{0} \Delta A=B_{0} \Delta B$. The angle $\theta=\arctan v / u$ is expanded in a Taylor series, and we get $\theta=\theta_{0}+\Delta \theta \cos k z$ with $\Delta \theta=\Delta B / A_{0}$. Hence $\Delta \theta$ should be comparable with $\Delta A_{v} /\left\langle A_{u}\right\rangle$. The value of the latter has the same order of magnitude as the former, but a bit smaller. In fact, the ratio $\left(\Delta A_{v} /\left\langle A_{u}\right\rangle\right) / \Delta \theta$ is about 0.7 for most solitons computed. We can think that the oscillations are partly due to the conservation of energy, but they are enhanced by the variations of the duration.

The mean value $\langle\theta\rangle$ of the angle that measures the optical field repartition between the two channels $u$ and $v$ is shown in Fig. 6. It is seen that $\langle\theta\rangle$ depends on the mean value $\left\langle A_{u}\right\rangle$ of the maximum amplitude of $u$, and varies in a quite small range, between about 14.5 degrees and 16 degrees. Also, we can notice from Figs. 5 and 6 that most part of the energy is contained in the $u$ component. However, the coupled system of Eqs. (1) and (2) remains unchanged if $u$ and $v$ are permuted. Hence a second family of solitons, for which most of energy is contained in the $v$ component, also exists and can be deduced from the above analysis by symmetry. Recall that the analytical soliton with $u= \pm v$ is unstable, as stated above.

\section{CONCLUSIONS}

We have analyzed the few-cycle vector optical solitons in two linearly coupled adjacent waveguides. We have considered the case of linear nondispersive coupling. The vector solitons feature specific oscillations with propagation distance $z$, which are the trace of the corresponding oscillations between two coupled modes that occur in the linear regime. However, strong discrepancies between linear and nonlinear propagation regimes are evidenced: In the nonlinear regime the range 
in which the ratio of the two field components of the fewcycle vector soliton oscillate is drastically reduced, and the frequency of oscillations is modified.

The knowledge of the stable states of the system allow us to interpret and even predict the outcome of the interaction of two incoming pulses in a waveguide coupler. Other types of waveguide coupling in the few-cycle regime, due to strong dispersion or nonlinearity, have also been discussed; see Ref. [55]. However, their effect on few-cycle vector soliton propagation will be reported elsewhere.
[1] T. Brabec and F. Krausz, Intense few-cycle laser fields: Frontiers of nonlinear optics, Rev. Mod. Phys. 72, 545 (2000).

[2] L. Gallmann, D. H. Sutter, N. Matuschek, G. Steinmeyer, U. Keller, C. Iaconis, and I. A. Walmsley, Characterization of sub6-fs optical pulses with spectral phase interferometry for direct electric-field reconstruction, Opt. Lett. 24, 1314 (1999).

[3] U. Morgner, F. X. Kärtner, S. H. Cho, Y. Chen, H. A. Haus, J. G. Fujimoto, E. P. Ippen, V. Scheuer, G. Angelow, and T. Tschudi, Sub-two-cycle pulses from a Kerr-lens mode-locked Ti:sapphire laser, Opt. Lett. 24, 411 (1999).

[4] D. H. Sutter, G. Steinmeyer, L. Gallmann, N. Matuschek, F. Morier-Genoud, U. Keller, V. Scheuer, G. Angelow, and T. Tschudi, Semiconductor saturable-absorber mirror assisted Kerr-lens mode-locked Ti:sapphire laser producing pulses in the two-cycle regime, Opt. Lett. 24, 631 (1999).

[5] A. Shirakawa, I. Sakane, M. Takasaka, and T. Kobayashi, Sub-5fs visible pulse generation by pulse-front-matched noncollinear optical parametric amplification, Appl. Phys. Lett. 74, 2268 (1999).

[6] M. Wegener, Extreme Nonlinear Optics (Springer, Berlin, 2005).

[7] M. Kolesik and J. V. Moloney, Modeling and simulation techniques in extreme nonlinear optics of gaseous and condensed media, Rep. Prog. Phys. 77, 016401 (2014).

[8] A. Nazarkin, Nonlinear Optics of Intense Attosecond Light Pulses, Phys. Rev. Lett. 97, 163904 (2006).

[9] F. Krausz and M. Ivanov, Attosecond physics, Rev. Mod. Phys. 81, 163 (2009).

[10] G. A. Mourou, N. J. Fisch, V. M. Malkin, Z. Toroker, E. A. Khazanov, A. M. Sergeev, T. Tajima, and B. Le Garrec, ExawattZettawatt pulse generation and applications, Opt. Commun. 285, 720 (2012).

[11] A. E. Kaplan, Beyond attoseconds, Lasers in Engineering 24, 3 (2013).

[12] G. Mourou, S. Mironov, E. Khazanov, and A. Sergeev, Single cycle thin film compressor opening the door to zeptosecondexawatt physics, Eur. Phys. J. Special Topics 223, 1181 (2014).

[13] Th. Brabec and F. Krausz, Nonlinear Optical Pulse Propagation in the Single-Cycle Regime, Phys. Rev. Lett. 78, 3282 (1997).

[14] Sh. Amiranashvili, U. Bandelow, and N. Akhmediev, Dispersion of nonlinear group velocity determines shortest envelope solitons, Phys. Rev. A 84, 043834 (2011).

[15] A. V. Husakou and J. Herrmann, Supercontinuum Generation of Higher-Order Solitons by Fission in Photonic Crystal Fibers, Phys. Rev. Lett. 87, 203901 (2001).

[16] Sh. Amiranashvili, U. Bandelow, and N. Akhmediev, Few-cycle optical solitary waves in nonlinear dispersive media, Phys. Rev. A 87, 013805 (2013).

[17] E. V. Kazantseva, A. I. Maimistov, and J.-G. Caputo, Reduced Maxwell-Duffing description of extremely short pulses in nonresonant media, Phys. Rev. E 71, 056622 (2005).
[18] N. N. Rosanov, V. V. Kozlov, and S. Wabnitz, Maxwell-DrudeBloch dissipative few-cycle optical solitons, Phys. Rev. A 81, 043815 (2010).

[19] S. V. Sazonov, On the nonlinear optics of few-cycle pulses, Bull. Russian Acad. Sciences 75, 157 (2011).

[20] H. Leblond, H. Triki, and D. Mihalache, Theoretical studies of ultrashort-soliton propagation in nonlinear optical media from a general quantum model, Rom. Rep. Phys. 65, 925 (2013).

[21] H. Leblond and D. Mihalache, Models of few optical cycle solitons beyond the slowly varying envelope approximation, Phys. Rep. 523, 61 (2013).

[22] D. J. Frantzeskakis, H. Leblond, and D. Mihalache, Nonlinear optics of intense few-cycle pulses: An overview of recent theoretical and experimental developments, Rom. J. Phys. 59, 767 (2014).

[23] I. V. Mel'nikov, D. Mihalache, F. Moldoveanu, and N.-C. Panoiu, Quasiadiabatic following of femtosecond optical pulses in a weakly excited semiconductor, Phys. Rev. A 56, 1569 (1997).

[24] T. Schäfer and C. E. Wayne, Propagation of ultra-short optical pulses in cubic nonlinear media, Physica D 196, 90 (2004).

[25] A. Sakovich and S. Sakovich, The short pulse equation is integrable, J. Phys. Soc. Jpn. 74, 239 (2005).

[26] J. C. Brunelli, The short pulse hierarchy, J. Math. Phys. 46, 123507 (2005).

[27] H. Leblond and F. Sanchez, Models for optical solitons in the two-cycle regime, Phys. Rev. A 67, 013804 (2003).

[28] H. Leblond, H. Triki, and D. Mihalache, Derivation of a generalized double sine-Gordon equation describing ultrashort soliton propagation in optical media composed of multilevel atoms, Phys. Rev. A 86, 063825 (2012).

[29] H. Leblond, S. V. Sazonov, I. V. Mel'nikov, D. Mihalache, and F. Sanchez, Few-cycle nonlinear optics of multicomponent media, Phys. Rev. A 74, 063815 (2006).

[30] H. Leblond and D. Mihalache, Few-optical-cycle solitons: Modified Korteweg-de Vries sine-Gordon equation versus other non-slowly varying envelope approximation models, Phys. Rev. A 79, 063835 (2009).

[31] S. P. Popov, Numerical analysis of soliton solutions of the modified Korteweg-de Vries-sine-Gordon equation, Comput. Math. Mathematical Phys. 55, 437 (2015).

[32] S. V. Sazonov, Extremely short and quasi-monochromatic electromagnetic solitons in a two-component medium, JETP 92, 361 (2001).

[33] V. G. Bespalov, S. A. Kozlov, Yu. A. Shpolyanskiy, and I. A. Walmsley, Simplified field wave equations for the nonlinear propagation of extremely short light pulses, Phys. Rev. A 66, 013811 (2002)

[34] S. A. Skobelev, D. V. Kartashov, and A. V. Kim, Few-OpticalCycle Solitons and Pulse Self-Compression in a Kerr Medium, Phys. Rev. Lett. 99, 203902 (2007). 
[35] S. V. Sazonov and N. V. Ustinov, Extremely short vector solitons under the conditions of conical refraction, JETP Lett. 99, 503 (2014).

[36] S. Amiranashvili, U. Bandelow, and N. Akhmediev, Ultrashort optical solitons in transparent nonlinear media with arbitrary dispersion, Opt. Quantum Electron. 46, 1233 (2014).

[37] A. A. Voronin and A. M. Zheltikov, Subcycle solitonic breathers, Phys. Rev. A 90, 043807 (2014).

[38] A. V. Kim, A. G. Litvak, V. A. Mironov, and S. A. Skobolev, Extreme self-compression along with superbroad spectrum upconversion of few-cycle optical solitons in the ionization regime, Phys. Rev. A 92, 033856 (2015).

[39] R. M. Arkhipov, M. V. Arkhipov, P. A. Belov, Yu. A. Tolmachev, and I. Babushkin, Generation of unipolar optical pulses in a Raman-active medium, Laser Phys. Lett. 13, 046001 (2016).

[40] D. V. Novitsky, Compression and collisions of chirped pulses in a dense two-level medium, Opt. Commun. 358, 202 (2016).

[41] C. L. Korpa, Gy. Tóth, and J. Hebling, Interplay of diffraction and nonlinear effects in the propagation of ultrashort pulses, J. Phys. B 49, 035401 (2016).

[42] S. V. Sazonov, Few-cycle solitons in the medium with permanent dipole moment under conditions of the induced birefringence, Opt. Commun. 380, 480 (2016).

[43] H. Leblond, D. Kremer, and D. Mihalache, Collapse of ultrashort spatiotemporal pulses described by the cubic generalized Kadomtsev-Petviashvili equation, Phys. Rev. A 81, 033824 (2010).

[44] H. Leblond, D. Kremer, and D. Mihalache, Ultrashort spatiotemporal optical solitons in quadratic nonlinear media: generation of line and lump solitons from few-cycle input pulses, Phys. Rev. A 80, 053812 (2009).

[45] B. A. Malomed, D. Mihalache, F. Wise, and L. Torner, Spatiotemporal optical solitons, J. Opt. B 7, R53 (2005).

[46] D. Mihalache, Linear and nonlinear light bullets: Recent theoretical and experimental studies, Rom. J. Phys. 57, 352 (2012).

[47] D. Mihalache, Multidimensional localized structures in optics and Bose-Einstein condensates: A selection of recent studies, Rom. J. Phys. 59, 295 (2014).

[48] D. Mihalache, Localized structures in nonlinear optical media: A selection of recent studies, Rom. Rep. Phys. 67, 1383 (2015).
[49] J. M. Dudley, G. Genty, and S. Coen, Supercontinuum generation in photonic crystal fiber, Rev. Mod. Phys. 78, 1135 (2006)

[50] A. M. Heidt, J. Rothhardt, A. Hartung, H. Bartelt, E. G. Rohwer, J. Limpert, and A. Tünnermann, High quality sub-two cycle pulses from compression of supercontinuum generated in allnormal dispersion photonic crystal fiber, Opt. Express 19, 13873 (2011).

[51] H. Leblond, Ph. Grelu, and D. Mihalache, Models for supercontinuum generation beyond the slowly-varying-envelope approximation, Phys. Rev. A 90, 053816 (2014).

[52] I. Babushkin, A. Husakou, J. Herrmann, and Y. S. Kivshar, Frequency-selective self-trapping and supercontinuum generation in arrays of coupled nonlinear waveguides, Opt. Express 15, 11978 (2007).

[53] A. V. Gorbach, W. Ding, O. K. Staines, C. E. de Nobriga, G. D. Hobbs, W. J. Wadsworth, J. C. Knight, D. V. Skryabin, A. Samarelli, M. Sorel, and R. M. De La Rue, Spatiotemporal nonlinear optics in arrays of subwavelength waveguides, Phys. Rev. A 82, 041802(R) (2010).

[54] H. Leblond and D. Mihalache, Linear and nonlinear waveguiding of few-cycle optical solitons in a planar geometry, Phys. Rev. A 88, 023840 (2013).

[55] H. Leblond and S. Terniche, Waveguide coupling in the fewcycle regime, Phys. Rev. A 93, 043839 (2016).

[56] R. Hirota, Bäcklund Transformations, the Inverse Scattering Method, Solitons, and Their Applications, Lecture Notes in Math. Vol. 515 (Springer, Berlin, 1976), pp. 40-68.

[57] S. C. Anco, N. Tchegoum Ngatat, and M. Willoughby, Interaction properties of complex modified Korteweg-de Vries (mKdV) solitons, Physica D 240, 1378 (2011).

[58] Yong Cheng, Zhen-Ya Yan, Biao Li, and Hong-Qing Zhang, New explicit exact solutions for a generalized Hirota-Satsuma coupled KdV system and a coupled mKdV equation, Chin. Phys. 12, 1 (2003).

[59] Bangqing Li and Yulan Ma, Exact solutions for coupled mKdV equations by a new symbolic computation method, Appl. Mech. and Mater. 20-23, 184 (2010).

[60] Yuan Gao and Xiao-Yan Tang, A coupled variable coefficient modified KdV equation arising from a two-layer fluid system, Commun. Theor. Phys. 48, 961 (2007). 